\title{
Long-term outcomes of endoscopic submucosal dissection for early gastric cancer: a retrospective comparison with conventional endoscopic resection in a single center
}

\author{
Satoshi Tanabe $\cdot$ Kenji Ishido $\cdot$ Katsuhiko Higuchi $\cdot$ \\ Tohru Sasaki $\cdot$ Chikatoshi Katada $\cdot$ Mizutomo Azuma $\cdot$ \\ Akira Naruke $\cdot$ Myungchul Kim $\cdot$ Wasaburo Koizumi
}

Received: 3 September 2012 / Accepted: 3 February 2013/Published online: 11 April 2013

(c) The International Gastric Cancer Association and The Japanese Gastric Cancer Association 2013

\begin{abstract}
Background Few studies have compared the outcomes of endoscopic mucosal resection (EMR) and endoscopic submucosal dissection (ESD) in patients with early gastric cancer.

Methods We studied 780 lesions for which endoscopic treatment was indicated according to the Japanese Gastric Cancer Association (JGCA) criteria or the extended National Cancer Center (NCC) criteria from April 1995 to December 2007. A total of 359 lesions were treated by endoscopic aspiration mucosectomy (EAM) between April 1995 and March 2003 (EAM group), and 421 lesions were treated by ESD between April 2003 and December 2007 (ESD group). Long-term outcomes (local recurrence rate, overall survival) were compared between the groups.

Results The median follow-up was 73 months in the EAM group and 65 months in the ESD group. Overall, the local recurrence rate was significantly lower in the ESD group $(0.2 \%, 1 / 421)$ than in the EAM group $(4.2 \%$, $15 / 359)(p<0.05)$. For lesions meeting the JGCA criteria, the local recurrence rate was $2.9 \%$ in the EAM group and $0 \%$ in the ESD group $(p<0.05)$. For lesions meeting the NCC criteria, the local recurrence rate was $12.5 \%$ in the EAM group and $0.6 \%$ in the ESD group $(p<0.05)$. There was no significant difference between the groups in overall survival.

Conclusions On long-term follow-up, ESD was associated with a lower rate of local recurrence than EAM for
\end{abstract}

S. Tanabe $\cdot$ K. Ishido $(\bowtie) \cdot$ K. Higuchi $\cdot$ T. Sasaki ·

C. Katada - M. Azuma - A. Naruke - M. Kim - W. Koizumi

Department of Gastroenterology, Kitasato University School

of Medicine, 2-1-1 Asamizodai, Minami-ku, Sagamihara,

Kanagawa 252-0380, Japan

e-mail: k.ishido@kitasato-u.ac.jp lesions that met the JGCA or the NCC criteria. From the point of view of radical curability, ESD can be recommended for the management of lesions that meet either set of criteria.

Keywords Early gastric cancer · Endoscopic aspiration mucosectomy $\cdot$ Endoscopic mucosal resection $\cdot$ Endoscopic submucosal dissection - Long-term outcomes

\section{Introduction}

In Japan, endoscopic resection is widely used to treat early gastric cancer because it allows preservation of the stomach, improving the quality of life of patients. Endoscopic mucosal resection (EMR) was initially performed by strip biopsy [1], followed by the development of techniques such as EMR with a cap-fitted panendoscope (EMRC) [2] and endoscopic aspiration mucosectomy (EAM) [3, 4]. In the latter half of the 1990s, techniques for endoscopic submucosal dissection (ESD) were developed and gained popularity [5, 6]. The advent of ESD has expanded the indication range for endoscopic resection [7]. Although ESD is technically challenging and can cause complications such as perforation, it has been reported to have a higher rate of en bloc resection than conventional EMR [8]. We previously reported that piecemeal resection has a slightly higher recurrence rate than en bloc resection in patients who undergo EMR for early gastric cancer [9]. However, studies comparing long-term rates of local recurrence between EMR and ESD are scant [10]. In the present study, we historically compared long-term local recurrence rates between conventional EAM and ESD performed with an insulation-tipped diathermic knife (IT Knife; Olympus Medical Systems, Tokyo, Japan). All 
procedures were performed in our department at a single center.

\section{Patients and methods}

Patients

A total of 852 consecutive lesions of early gastric cancer treated by endoscopic resection between April 1995 and December 2007 were studied. Informed consent was obtained from all patients in accordance with our institutional protocol. According to the Japanese Gastric Cancer Association (JGCA) criteria, endoscopic resection is indicated for the treatment of differentiated mucosal cancer measuring less than $2 \mathrm{~cm}$ in diameter, without ulceration [7]. On the other hand, the expanded National Cancer Centre (NCC) criteria proposed by Gotoda et al. [11] recommend endoscopic resection for the management of differentiated mucosal cancer regardless of size in the absence of ulceration or less than $3 \mathrm{~cm}$ in diameter in the presence of ulceration. In addition, we included lesions with minute submucosal invasion (SM1: invasion depth $<500 \mu \mathrm{m}$ below the muscularis mucosae) that were less than $30 \mathrm{~mm}$ in diameter.

Seventy-two of the consecutive 852 early gastric cancers did not satisfy either criterion for endoscopic resection and were excluded. The remaining 780 lesions were divided into two groups: 359 lesions treated by EAM between April 1995 and March 2003 (EAM group) and 421 lesions treated by ESD between April 2003 and December 2007 (ESD group).

\section{Methods}

\section{Diagnosis}

Patients underwent esophagogastroduodenoscopy (EGD) and abdominal computed tomographic scanning as pretreatment examinations. Endoscopic ultrasonography was performed as required.

\section{Resection technique}

\section{EAM}

EAM was performed as described by Torii et al. [3]. Briefly, the lesion margin was marked with a heater probe. A solution of $10 \%$ glycerin plus fructose $(10 \%$ glycerol; Chugai Pharmaceutical, Tokyo, Japan) was then injected via an injection needle at the lesion margin to elevate the lesion. The endoscope was inserted through an overtube to ensure safety. An aspiration mucosector (Top Co., Tokyo, Japan) was attached to the tip of a conventional endoscope. The endoscope with the aspiration mucosector was then inserted, and the lesion was aspirated, using the previously marked margin for reference. The snare was opened, the lesion was ensnared, leaving about $1 \mathrm{~cm}$ of tissue protruding from the snare, and electrosurgical current was applied to resect the lesion.

Endoscopic submucosal dissection

The circumference of the lesion was marked with a needle knife. After injecting glycerol solution into the submucosa, an initial cut was made with a needle knife outside the marking. An IT Knife (Olympus Medical Systems) was inserted into this cut and operated to cut around the lesion [5]. The marked lesion was separated from the surrounding normal mucosa. Then, the submucosal layer was dissected using the IT Knife, and the lesion was finally removed. An IT Knife was used to perform ESD until the end of March 2007, and an IT Knife2 (Olympus Medical Systems) was then used from April 2007 onward [12]. If resection was incomplete, additional treatment was performed, mainly by thermocoagulation therapy (laser, heater probe, or argon plasma coagulation).

\section{Follow-up}

All patients whose lesions met JGCA criteria were followed up by annual upper gastrointestinal endoscopy to detect local recurrence and metachronous gastric cancer. Patients who met the NCC criteria were additionally followed up by abdominal ultrasound or computed tomography every 6 months to detect metastatic lesions.

\section{Histopathological evaluation}

The tissue specimens were fixed in formalin, cut into 2-mm-wide strips perpendicular to the lesion base, and embedded in paraffin. A pathologist examined the sections to determine the histopathological diagnosis according to the Japanese Classification of Gastric Carcinoma [13]. Tumor size, depth of invasion, presence of ulcerative changes, lymphatic and vascular involvement, and tumor involvement of the lateral and vertical margins were assessed. The depth of invasion was defined as follows: M, mucosal invasion; SM1, minute submucosal invasion $(<500 \mu \mathrm{m}$ below the muscularis mucosae); and SM2, submucosal invasion $(\geq 500 \mu \mathrm{m}$ below the muscularis mucosae). 
En bloc resection was defined as the endoscopic resection of an entire lesion in a single procedure. Complete resection was defined as the endoscopic resection of an entire lesion in a single procedure, with no histopathological evidence of tumor at the resection margin. Incomplete resection was defined as tumor-positive margins on histopathological examination. Endoscopically resected lesions with margins that could not be evaluated histopathologically because of electrosurgical or mechanical damage were classified as not assessable.

\section{Statistical analysis}

Survival time was calculated as interval between the date of the first session of EAM or ESD and the date of death or the last date confirmed as alive for survivors. Survival curves were calculated using the Kaplan-Meier method. The log-rank test was used to compare survival. All $p$ values reported are two sided, and $p<0.05$ was considered to indicate statistical significance.

\section{Results}

Table 1 shows the patient and tumor characteristics. As for the patients' characteristics, the sex ratio did not differ significantly between the treatment groups, but mean age was significantly higher in the ESD group (69 years) than in the EAM group (67 years; $p<0.05)$. As for tumor characteristics, 311 lesions $(86.6 \%)$ met the JGCA criteria and $48(13.4 \%)$ met the NCC criteria in the EAM group, as compared with 262 lesions $(62.2 \%)$ that met the JGCA criteria and $159(37.8 \%)$ that met the NCC criteria in the ESD group. The proportion of lesions that met the NCC criteria was significantly higher in the ESD group $(p<0.05)$. The size of tumors that met the JGCA criteria did not differ between the groups. The size of tumors that met the NCC criteria was significantly greater in the ESD group than in the EAM group. Tumor location did not differ significantly between the groups, but the proportion of tumors macroscopically classified as depressed and/or flat type was significantly higher in the ESD group $(p<0.05)$.

\section{Short-term outcomes}

Table 2 shows early outcomes. Operation time was significantly longer in the ESD group $(67 \mathrm{~min})$ than in the EAM group $(30 \mathrm{~min})(p<0.05)$. The rate of complete resection (en bloc and tumor-free margin resection) was significantly higher in the ESD group (92.9\%, 391/421) than in the EAM group $(52.1 \%, 187 / 359)(p<0.05)$. As for complications, the incidences of delayed bleeding and perforation did not differ significantly between the groups.

Long-term outcomes

Table 3 compares local recurrence between the groups. Among lesions that satisfied the JGCA criteria, the rate of local recurrence was significantly higher in the EAM group $(2.9 \%, 9 / 311)$ than in the ESD group (0\%, 0/262; $p<0.05)$. The rate of local recurrence among lesions that met the NCC criteria (i.e., the expanded indication range

Table 1 Patient and tumor characteristics

\begin{tabular}{|c|c|c|c|}
\hline & $\begin{array}{l}\text { Endoscopic } \\
\text { aspiration } \\
\text { mucosectomy } \\
(\text { EAM) group } \\
(n=359)\end{array}$ & $\begin{array}{l}\text { Endoscopic } \\
\text { submucosal } \\
\text { dissection }(\mathrm{ESD}) \\
\text { group }(n=421)\end{array}$ & $p$ value \\
\hline $\operatorname{Sex}(\mathrm{MF})$ & $246 / 73$ & $276 / 95$ & NS \\
\hline $\begin{array}{l}\text { Mean age, } \\
\text { years }( \pm \mathrm{SD})\end{array}$ & $67 \pm 9.6(38-90)$ & $69 \pm 8.6(41-91)$ & $<0.05$ \\
\hline \multicolumn{4}{|c|}{ Tumor indication criteria } \\
\hline $\begin{array}{l}\text { JGCA } \\
\text { (Japanese } \\
\text { Gastric } \\
\text { Cancer } \\
\text { Association) }\end{array}$ & 311 & 262 & $<0.05$ \\
\hline $\begin{array}{l}\text { NCC } \\
\text { (National } \\
\text { Cancer } \\
\text { Center) }\end{array}$ & 48 & 159 & \\
\hline \multicolumn{4}{|c|}{ Median tumor size $(\mathrm{mm})$} \\
\hline JGCA & $11(1-20)$ & $11(1-20)$ & NS \\
\hline $\mathrm{NCC}$ & $12(2-30)$ & $24(2-71)$ & $<0.05$ \\
\hline \multicolumn{4}{|l|}{ Tumor location } \\
\hline Upper third & 85 & 105 & NS \\
\hline Middle third & 115 & 140 & \\
\hline Lower third & 159 & 176 & \\
\hline \multicolumn{4}{|c|}{ Macroscopic type } \\
\hline Elevated & 190 & 152 & $<0.05$ \\
\hline $\begin{array}{l}\text { Depressed } \\
\text { and/or flat }\end{array}$ & 169 & 269 & \\
\hline
\end{tabular}

Table 2 Early outcomes of EAM and ESD

\begin{tabular}{|c|c|c|c|}
\hline & $\begin{array}{l}\text { EAM group } \\
(n=338)\end{array}$ & $\begin{array}{l}\text { ESD group } \\
(n=397)\end{array}$ & $p$ value \\
\hline Operation time & $30 \min (5-115)$ & $67 \min (7-360)$ & $<0.05$ \\
\hline $\begin{array}{l}\text { Delayed } \\
\text { bleeding }\end{array}$ & $12 / 338(3.6 \%)$ & $25 / 397(6.3 \%)$ & NS \\
\hline Perforation & $11 / 338(3.3 \%)$ & $13 / 397(3.3 \%)$ & NS \\
\hline \multicolumn{4}{|l|}{ Curability } \\
\hline $\begin{array}{l}\text { Complete } \\
\text { resection }\end{array}$ & $187 / 359(52.1 \%)$ & $391 / 421(92.9 \%)$ & $<0.05$ \\
\hline
\end{tabular}


Table 3 Comparison of local recurrence

\begin{tabular}{lclc}
\hline & $\begin{array}{l}\text { EAM group } \\
(n=359)\end{array}$ & $\begin{array}{l}\text { ESD group } \\
(n=421)\end{array}$ & $p$ value \\
\hline $\begin{array}{l}\text { JGCA criteria } \\
(\%)\end{array}$ & $9 / 311(2.9)$ & $0 / 262(0)$ & $<0.05$ \\
$\begin{array}{l}\text { NCC criteria } \\
(\%)\end{array}$ & $6 / 48(12.5)$ & $1 / 159(0.6)$ & $<0.05$ \\
Total & $15 / 359(4.2)$ & $1 / 421(0.2)$ & $<0.05$ \\
\hline
\end{tabular}

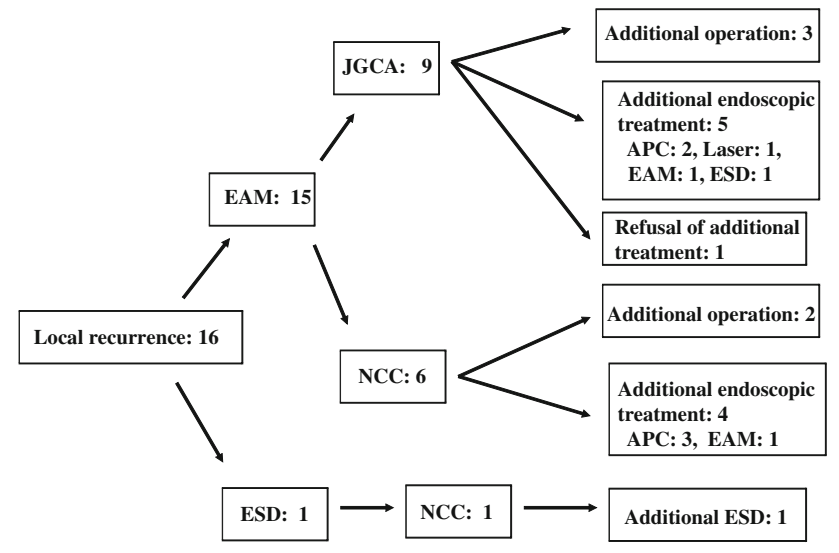

Fig. 1 Additional treatment for local recurrence

for endoscopic resection) was significantly higher in the EAM group $(12.5 \%, 6 / 48)$ than in the ESD group $(0.6 \%$, $1 / 159 ; p<0.05)$. The overall rate of local recurrence was also significantly higher in the EAM group $(4.2 \%, 15 / 359)$ than in the ESD group $(0.2 \%, 1 / 421 ; p<0.05)$. The median follow-up was 73 months in the EAM group and 65 months in the ESD group.

Additional treatments given to the 16 patients who had local recurrence are shown in Fig. 1. Fifteen of the patients belonged to the EAM group. Among the 9 patients with lesions that met the JGCA criteria, 3 underwent additional surgery, 5 received additional endoscopic treatment, and 1 refused additional therapy and was observed. Among the 6 patients with lesions that satisfied the NCC criteria, 2 underwent additional surgery, and 4 received additional endoscopic treatment. In the ESD group, only 1 patient received additional treatment (additional ESD). This patient had a lesion that met the NCC criteria. The time to recurrence was 5 months in the 1 patient with recurrence in the ESD group. The median time to recurrence was 14 months (range 2-35 months) in the 15 patients with recurrence in the EAM group. Metastatic recurrence occurred in only 1 patient. This patient received ESD for a tumor that met the NCC criteria. There was no metastatic recurrence in the EAM group.

The cumulative residual tumor-free/recurrence-free curves are shown for patients with lesions that met the JGCA criteria, patients with lesions that met the NCC

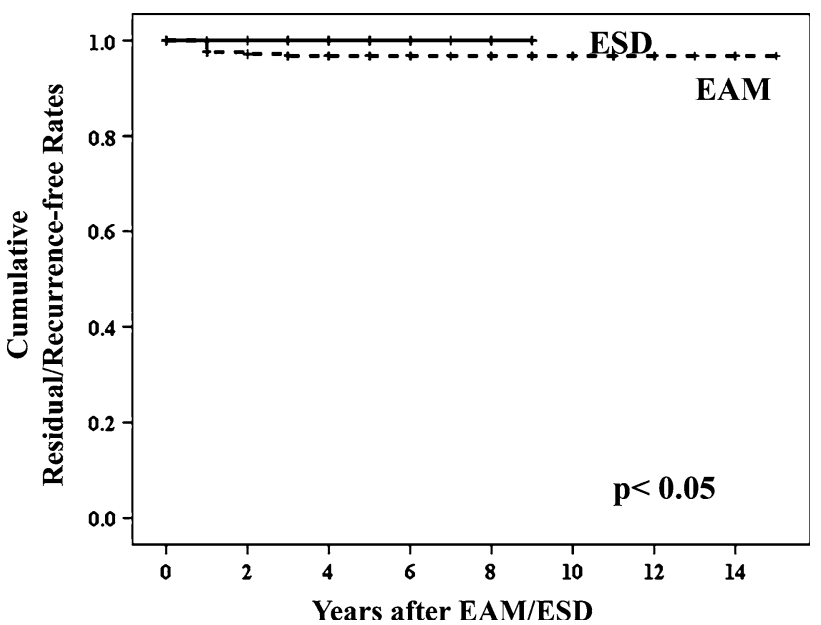

Fig. 2 Cumulative residual tumor-free/recurrence-free curves for patients whose tumors met the Japanese Gastric Cancer Association (JGCA) criteria $(n=573)$

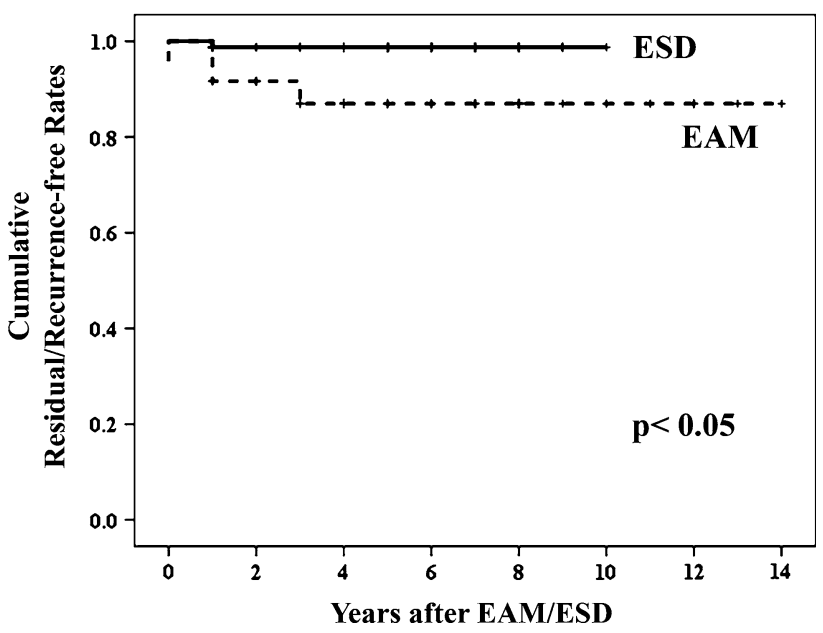

Fig. 3 Cumulative residual tumor-free/recurrence-free curves for patients whose tumors met the National Cancer Center (NCC) criteria $(n=207)$

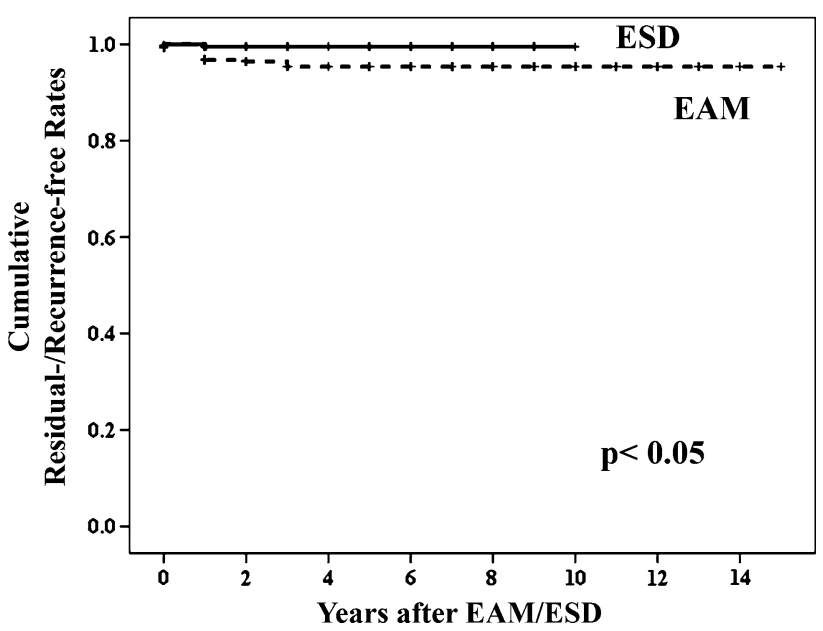

Fig. 4 Cumulative residual tumor-free/recurrence-free curves for the study group as a whole $(n=780)$ 


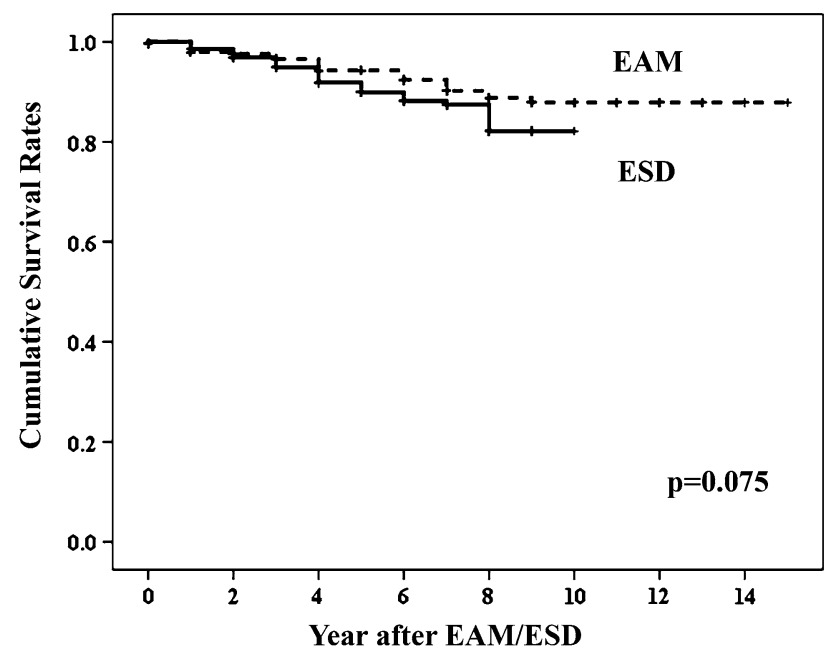

Fig. 5 Cumulative overall survival curves

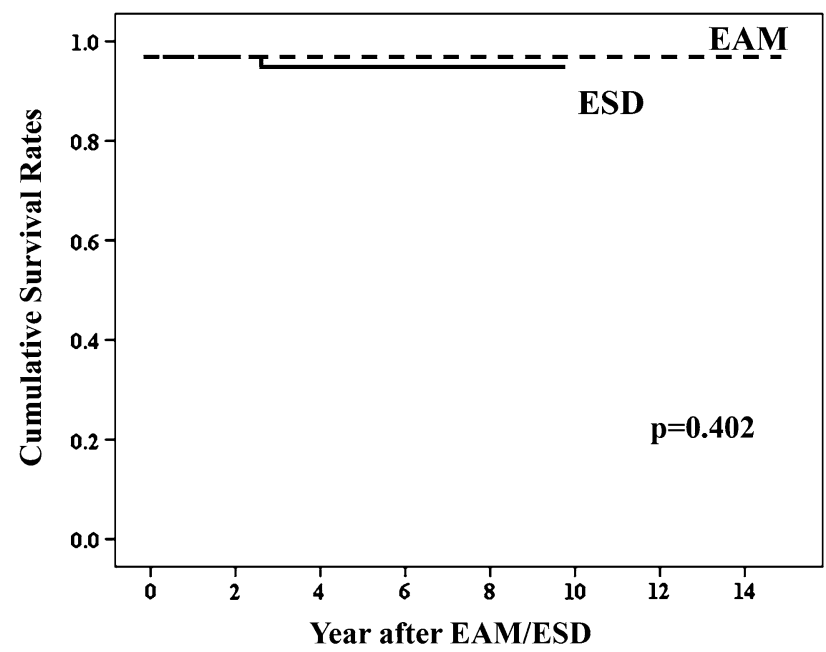

Fig. 6 Cumulative overall survival curves after exclusion of deaths from causes unrelated to gastric cancer

criteria, and for the study group as a whole in Figs. 2, 3, and 4 , respectively. At 3 years, the residual tumor-free/ recurrence-free rates were significantly higher in the ESD group than in the EAM group among patients whose lesions met the JGCA criteria (100 vs. $97.4 \% ; p<0.05$ ), among patients whose lesions met the NCCA criteria ( 98.7 vs. $86.9 \% ; p<0.05)$, and among the study groups as a whole ( 99.5 vs. $98.2 \% ; p<0.05)$. At 5 years, the residual tumor-free/recurrence-free rates were also significantly higher in the ESD group than in the EAM group among patients whose lesions met the JGCA criteria (100 vs. $97.0 \% ; p<0.05$ ), among patients whose lesions met the NCCA criteria (98.7 vs. $86.9 \% ; p<0.05$ ), and among the study group as a whole (99.5 vs. $96.6 \% ; p<0.05)$.

The overall survival curves are shown in Figs. 5 and 6. When patients who died of all causes were included, there was a slight trend toward better overall survival in the EAM group than in the ESD group. However, when only gastric cancer-related deaths were included in the calculation of overall survival, only one patient died of gastric cancer in the ESD group, with no gastric cancer-related deaths in the EAM group. There was no difference between the groups. In the patient who died, the primary lesion satisfied the NCC criteria, and lymph node recurrence developed 14 months after ESD. Additional operation was performed, but the patient died of gastric cancer 34 months after ESD. The histopathological diagnosis was poorly differentiated mixed-type cancer.

\section{Discussion}

More than 25 years have elapsed since the development of EMR, and the subsequent advent of ESD led to the even wider acceptance of endoscopic therapy as a minimally invasive procedure for the treatment of early gastric cancer. The major advantage of ESD is the ability to resect large and ulcerative lesions en bloc, and the rate of en bloc complete resection is higher than that of EMR $[6,8]$. En bloc resection facilitates the correct pathological evaluation of lesions. However, few studies have compared long-term outcomes between EMR and ESD. In the present study, we historically compared long-term local recurrence between EMR (EAM) and ESD.

Several studies have assessed local recurrence rates after EMR and ESD performed at single centers. Ryu et al. [14] reported recurrence rates of 9.6 and $3.5 \%$ in patients with gastric adenoma or early gastric cancer who underwent conventional EMR and ESD, respectively. Park et al. [10] reported recurrence rates of $18 \%$ for conventional EMR and $3.7 \%$ for ESD in patients with early gastric cancer. These studies found that the local recurrence rate was significantly higher for EMR than for ESD in patients treated at a single center. Multicenter clinical trials have also shown that ESD has lower rates of local residual tumor and recurrence than EMR [15]. Tada et al. [1] reported local recurrence in $6(6.9 \%)$ of 87 patients with early gastric cancer who underwent EMR by strip biopsy. All 6 patients with local recurrence had undergone incomplete resection.

Our department introduced strip biopsy [1] as the basic procedure for EMR of early gastric cancer in 1986. Subsequently, we gradually switched to EAM because the procedure is straightforward and enables the resection of larger specimens than does strip biopsy [4]. However, conventional procedures for EMR such as EAM have a risk of recurrence because of incomplete resection when piecemeal EMR is used to resect larger lesions [8]. We consequently started to perform ESD in April 2003 because 
of its higher rate of complete en bloc resection. We concurrently use ESD to resect lesions that meet either the JGCA or NCC criteria.

In the present study, the local recurrence rate was significantly lower in the ESD group $(0.2 \%, 1 / 421)$ than in the EAM group $(4.2 \%, 15 / 359)(p<0.05)$. We also evaluated local recurrence according to whether lesions met the JGCA criteria or NCC criteria for endoscopic resection. Among lesions that satisfied the JGCA criteria, the rate of local recurrence was significantly lower in the ESD group $(0 \%)$ than in the EAM group $(2.9 \%)$. It is noteworthy that no patient in the ESD group had local recurrence.

The rate of local recurrence among lesions that satisfied the NCC criteria defining the expanded indications for endoscopic resection was $12.5 \%$ in the EAM group as compared with only $0.95 \%$ in the ESD group. Although lesions that met NCC criteria were significantly larger in the ESD group than in the EAM group, the recurrence rate was clearly lower for ESD than for conventional EMR. Our findings suggest that ESD can be recommended for lesions that satisfy either the JGCA or NCC criteria. The time to recurrence was 5 months in the 1 patient with recurrence in the ESD group. In the 15 patients with recurrence in the EAM group, the median time to recurrence was 14 months, ranging from 2 to 35 months. All cases of local recurrence were detected within 3 years in both groups, indicating the need for close follow-up during the first 3 years after treatment.

Long-term overall survival is generally similar for EAM as well as ESD. However, EAM has a higher rate of local recurrence, requiring additional endoscopic or surgery therapy. In contrast, ESD has an extremely low rate of local recurrence as well as a higher curative resection rate. We therefore recommend ESD for the management of early gastric cancer that meets either the JGCA or NCC criteria. ESD is expected to replace EAM as a standard technique for the endoscopic treatment of early gastric cancer.

Nodal recurrence developed after ESD in one patient who had a poorly differentiated mixed-type intramucosal cancer that satisfied the NCC criteria [16]. We previously studied the feasibility of ESD in a patient with a poorly differentiated mixed-type cancer with submucosal invasion and reported that such mixed histological type cancers were associated with a higher risk of lymph node metastasis than "pure" poorly differentiated cancers [17]. Caution is therefore required in patients with poorly differentiated mixed-type cancers.

Gotoda et al. [10] studied long-term outcomes after ESD and found that the outcomes of patients with lesions that met the NCC criteria were similar to those of patients whose lesions met the guideline (JGCA) criteria. ESD had good outcomes in terms of the en bloc resection rate of large lesions, as well as a very low recurrence rate.
ESD is generally considered necessary for the resection of large lesions or lesions accompanied by ulceration, but this is prospectively being confirmed in an ongoing study in patients with gastric cancer meeting the NCC criteria, conducted primarily by the Japan Clinical Oncology Group (JCOG).

In summary, our results show that ESD is associated with a significantly lower rate of local recurrence than EAM for early gastric cancer that meets either the JGCA criteria or NCC criteria. From the point of view of radical curability, ESD can be recommended for the management of lesions that meet either set of criteria.

Conflict of interest None.

\section{References}

1. Tada M, Murakami A, Karita M, et al. Endoscopic resection of early gastric cancer. Endoscopy. 1993;25:445-50.

2. Inoue $\mathrm{H}$, Takeshita $\mathrm{K}$, Hori $\mathrm{H}$, et al. Endoscopic mucosal resection with a cap-fitted panendoscope for esophagus, stomach, and colon mucosal lesions. Gastrointest Endosc. 1993;39:58-62.

3. Torii A, Sakai M, Kajiyama T, et al. Endoscopic aspiration mucosectomy as curative endoscopic surgery; analysis of 24 cases of early gastric cancer. Gastrointest Endosc. 1995;42: 475-9.

4. Tanabe S, Koizumi W, Kokutou M, et al. Usefulness of endoscopic aspiration mucosectomy as compared with strip biopsy for the treatment of gastric mucosal cancer. Gastrointest Endosc. 1999;50:819-22.

5. Ohkuwa M, Hosokawa K, Boku N, et al. New endoscopic treatment for intramucosal gastric tumors using an insulated-tip diathermic knife. Endoscopy. 2001;33:221-6.

6. Ono $\mathrm{H}$, Kondo H, Gotoda T, et al. Endoscopic mucosal resection for treatment of early gastric cancer. Gut. 2001;48:225-9.

7. Gotoda T, Yanagisawa A, Sasako M, et al. Incidence of lymph node metastasis from early gastric cancer: estimation with a large number of cases at two large centers. Gastric Cancer 2000; 3(4):219-225.

8. Watanabe K, Ogata S, Kawazoe S, et al. Clinical outcomes of EMR for gastric tumors: historical pilot evaluation between endoscopic submucosal dissection and conventional mucosal resection. Gastrointest Endosc. 2006;63:776-82.

9. Tanabe S, Koizumi W, Mitomi H, et al. Clinical outcome of endoscopic aspiration mucosectomy for early gastric cancer. Gastrointest Endosc. 2002;56:708-13.

10. Park JC, Lee SK, Seo JH, et al. Predictive factors for local recurrence after endoscopic resection for early gastric cancer: long-term clinical outcome in a single-center experience. Surg Endosc. 2010;24:2842-9.

11. Gotoda T, Iwasaki M, Kusano C, et al. Endoscopic resection of early gastric cancer treated by guideline and expanded National Cancer Centre criteria. Br J Surg. 2010;97:868-71.

12. Ono H, Hasuike N, Inui $\mathrm{T}$, et al. Usefulness of a novel electrosurgical knife, the insulation-tipped diathermic knife-2, for endoscopic submucosal dissection of early gastric cancer. Gastric Cancer. 2008;11:47-52.

13. Japanese Gastric Cancer Association. Japanese classification of gastric carcinoma, 3rd English edition. Gastric Cancer. 2011;14: $101-12$. 
14. Ryu CB, Kim SG, Jung SW, et al. The usefulness and limitation of endoscopic mucosal resection using the incision and submucosal dissection methods (EISD) for early gastric cancer and gastric flat adenoma in Korea. Gastrointest Endosc 2005;61: $\mathrm{AB} 238$

15. Oda I, Gotoda T, Hamanaka H, et al. Endoscopic submucosal resection for early gastric cancer: technical feasibility, operation time and complications from a large consecutive series. Dig Endosc. 2005; 17:54-8.
16. Hanaoka N, Tanabe S, Higuchi K, et al. A rare case of histologically mixed-type intramucosal gastric cancer accompanied by nodal recurrence and liver metastasis after endoscopic submucosal dissection. Gastrointest Endosc. 2009;69:588-90.

17. Hanaoka N, Tanabe S, Mikami T, et al. Mixed-histologic-type submucosal invasive gastric cancer as a risk factor for lymph node metastasis: feasibility of endoscopic submucosal dissection. Endoscopy. 2009;41:427-32. 